\title{
Electrostric tive nonlinearity in optical fiber deduced from Brillouin gain measurements
}

\author{
Luc Thévenaz, Alain Kueng, Marc Niklès, Philippe Robert \\ EPFL Swiss Federal Institute of Technology \\ Metrology Laboratory \\ 1015 Lausanne \\ Switzerland
}

\begin{abstract}
Brillouin gain spectrum measurements make possible a quantitative evaluation of the electrostrictive contribution to the optical Kerr effect at frequencies corresponding to picosecond pulses. It turns out that this contribution is much larger than formerly estimated.
\end{abstract}

\section{Summary}

The nonlinear refractive index has drawn considerable attention these past few years, since it may significantly perturbate long-range propagation of lightwaves in optical fibers. Two main origins to this nonlinearity have been identified ${ }^{1}$ : non resonant electronic nonlinearity and electrostriction. These contributions differently interact with light, so that it is of prime importance to quantify the relative importance of each contribution. Some recent works demonstrated that 
electrostriction may contribute more significantly than formerly expected ${ }^{2}$ and a tentative quantification was even reported ${ }^{3}$.

On the other hand the electrostriction is the main driving mechanism of stimulated Brillouin scattering that is today quantatively very well described, since very accurate measurements of the Brillouin linear gain $g_{B}$ have been recently obtained ${ }^{4}$. This paper shows that the electrostrictive contribution to the nonlinear refractive index $n_{2}$ may indeed be straightforwardly calculated from Brillouin gain measurements.

The Brillouin linear gain $g_{B}$ is dependent on material properties and in particular on the electrostrictive coefficient $\gamma_{e}=\rho \partial \varepsilon / \partial \rho$, according to the following relation':

$$
g_{B}=\frac{2 \pi \gamma_{e}^{2}}{c_{o} \varepsilon_{o}^{2} \lambda_{o}^{2} \rho \Delta v_{B} c_{A} n}
$$

where $c_{o}$ is the vacuum light velocity, $\lambda_{o}$ the vacuum wavelength, $\rho$ the fiber material density, $\Delta v_{B}$ the Brillouin FWHM linewidth, $n$ the refractive index and $c_{A}$ the fibre acoustic velocity that is directly deduced from the Brillouin Stokes shift $v_{B}=2 n c_{A} / \lambda_{0}$. The three parameters $g_{B}, v_{B}$ and $\Delta v_{B}$ fully characterize the Brillouin gain spectrum and were measured with a high accuracy ${ }^{4}$, as shown in Fig. 1. The electrostrictive coefficient $\gamma_{e}$ may be easily calculated from the gain spectrum measurements, since the other material coefficients are well known. 
The electrostrictive pressure is balanced by the medium compression, resulting in an increased density and thus a modified susceptibility ${ }^{1}$ :

$$
\Delta \chi=\frac{\Delta \varepsilon}{\varepsilon_{0}}=\frac{1}{2} \frac{C \gamma_{e}^{2}}{\varepsilon_{o}} \frac{|E|^{2}}{2}
$$

where $C=\rho \partial p / \partial \rho$ is the medium compressibility and $|E|^{2} / 2$ results from the timeaveraging of the squared optical field $E^{2}$. The electrostriction generates a susceptibility change proportional to the optical intensity $I=\left(n \varepsilon_{o} c_{0} / 2\right)|E|^{2}$, that modifies the refractive index this way:

$$
\Delta n=\frac{\partial n}{\partial \chi} \Delta \chi=\frac{\Delta \chi}{2 n}=\frac{1}{4} \frac{C \gamma_{e}^{2}}{\varepsilon_{o}}|E|^{2}=\frac{1}{4} \frac{C \gamma_{e}^{2}}{n^{2} \varepsilon_{o}^{2} c_{o}} I
$$

so that the electrostrictive nonlinear refractive index reads:

$$
n_{2}^{\text {elec }}=\frac{C \gamma_{e}^{2}}{4 n^{2} \varepsilon_{o}^{2} c_{o}}=0.7 \times 10^{-20} \frac{\mathrm{m}^{2}}{\mathrm{~W}} \quad \text { for a standard single mode fiber. }
$$

Assuming a total nonlinear refractive index $n_{2}=2.96 \times 10^{-20} \mathrm{~m}^{2} / \mathrm{W}$ the contribution of the electrostriction to the Kerr effect amounts to $23 \%$ at a $12-13 \mathrm{GHz}$ frequency, corresponding to pulse width in the $20-30$ ps range. This is much more in magnitude and in bandwidth than what was formerly suggested ${ }^{3}$, since the maximal calculated value of $16 \%$ was expected to rapidly vanish for pulse width narrower than $1 \mathrm{~ns}$. 
Fig. 2 shows the electrostrictive contribution to the nonlinear refractive index deduced from Brillouin gain measurements for a wide bunch of fibers having different $\mathrm{GeO}_{2}$ core doping concentration. The scattering of the results is most probably due to an unknown factor specific to stimulated Brillouin scattering: the overlapping degree between optical and acoustic guided modes. This factor is always smaller than unity, so that the electrostrictive contribution is at most underestimated using Brillouin gain measurements. The actual value should be close to $50 \%$ of the total nonlinear index, taking into account an estimation of the overlapping degree.

1 R. W. Boyd, "Nonlinear Optics", Ch. 4, Academic Press, inc., 1992, San Diego, CA, USA

2 E. Dianov, A. Luchnikov, A. Pilipetskii and A. Starodumov, Opt. Lett., 15, p. 314, (1990)

3. E. L. Buckland, R.W. Boyd, OFC'96 Technical digest, OSA publications, ThF2, pp. 224-225, (1996)

4 M. Niklès, L. Thévenaz, Ph. Robert, J. Light. Tech., 15, October 1997, in press 


\section{Figure captions}

Fig 1 High-accuracy Brillouin gain spectrum measurement of a single mode silica fiber performed at a $1319 \mathrm{~nm}$ wavelength, with the estimated value of the three characterizing parameters.

Fig. 2 Calculated electrostrictive contribution to the nonlinear refractive index from Brillouin gain spectrum measured in single mode fibers with different core doping. 

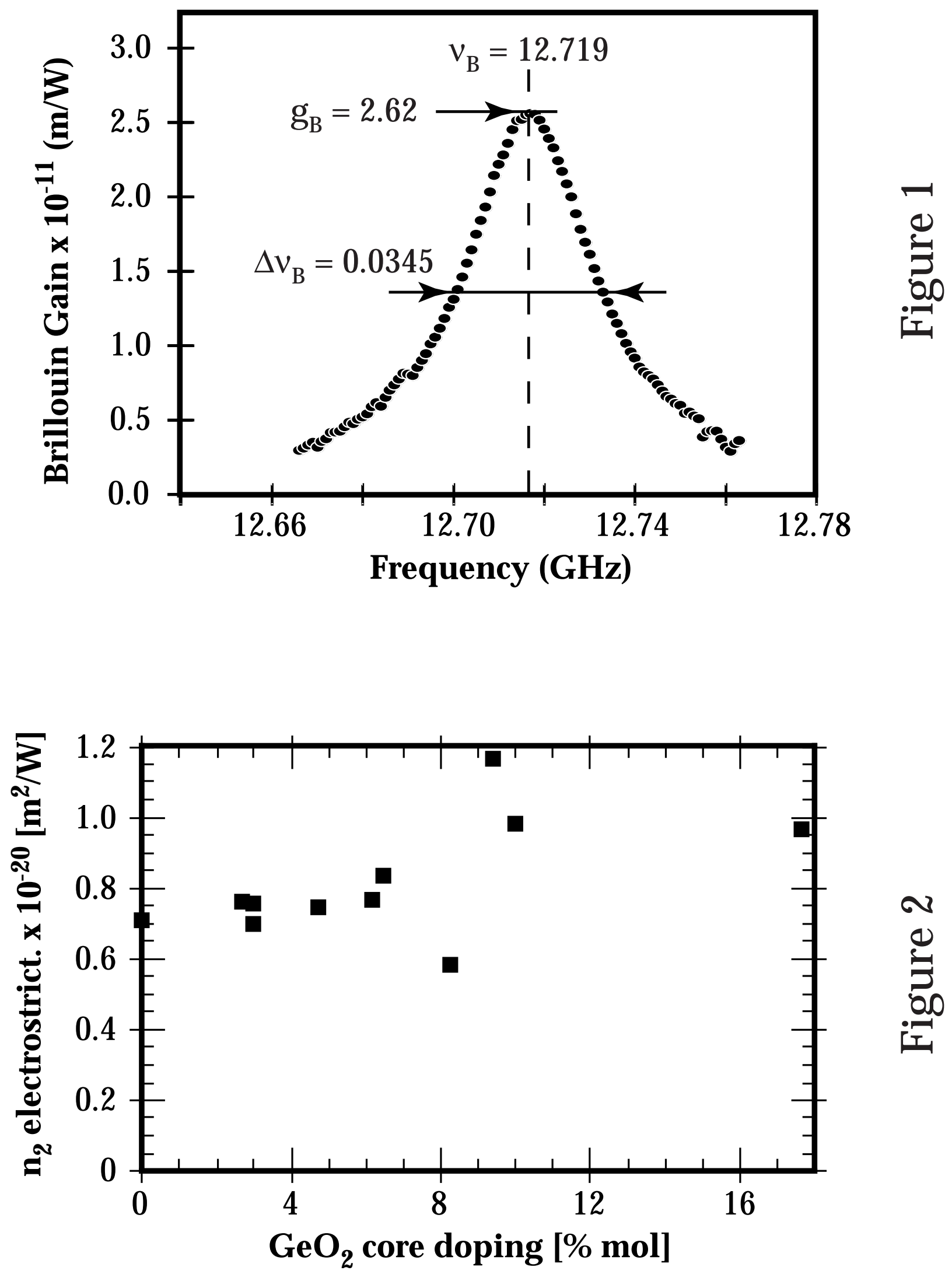